\title{
Selective negative-ion formation from core-valence doubly excited states of the water molecule
}

\author{
Christian Stråhlman* \\ Department of Materials Science and Applied Mathematics, Malmö University, 20506 Malmö, Sweden, \\ and MAX IV Laboratory, Lund University, P.O. Box 118, 22100 Lund, Sweden \\ Bruno Moog \\ Optoelectronics Research Centre, University of Southampton, Highfield Campus, Hampshire, SO17 1BJ, United Kingdom, \\ and MAX IV Laboratory, Lund University, P.O. Box 118, 22100 Lund, Sweden \\ Emelie Ertan and Michael Odelius \\ Department of Physics, Stockholm University, AlbaNova University Center, 10691 Stockholm, Sweden \\ Antti Kivimäki \\ Nano and Molecular Systems Research Unit, University of Oulu, P.O. Box 3000, 90014 Oulu, Finland, \\ and MAX IV Laboratory, Lund University, P.O. Box 118, 22100 Lund, Sweden \\ Robert Richter \\ Elettra-Sincrotrone Trieste, Area Science Park, 34149 Trieste, Italy \\ Rami Sankari \\ MAX IV Laboratory, Lund University, P.O. Box 118, 22100 Lund, Sweden
}

(Received 13 September 2018; published 26 November 2018)

\begin{abstract}
This study focuses on the role of negative-ion formation in the decay of core-valence doubly excited water molecules. Combining negative- and positive-ion coincidence measurements with calculated energies of corevalence doubly excited states, we find that $\mathrm{O}^{-}$and $\mathrm{H}^{-}$production is enhanced selectively. In particular, we suggest that $\mathrm{O}^{-}$production is correlated to double occupancy of the antibonding $4 a_{1}$ virtual orbital, while $\mathrm{H}^{-}$ appears at electronic states with double occupancy in $2 b_{2}$. We also show that $\mathrm{H}^{-}$and $\mathrm{O}^{-}$can be created as a result of electron recapture close to the $\mathrm{O} 1 s$ ionization potential.
\end{abstract}

DOI: 10.1103/PhysRevA.98.053432

\section{INTRODUCTION}

Water is probably the most studied compound, and photoninduced dissociation has been the topic of several earlier works. Nevertheless, despite the apparent simplicity of the water molecule, several things remain unknown about it. Studies of the fragmentation of the water molecule provide insights into the molecule's constitution and chemical properties. Photodissociation of water is a process commonly studied in order to establish an efficient hydrogen-production method for energy generation and storage or to improve our knowledge of atmospheric chemistry. A comprehensive understanding of the fragmentation process is fundamental to progress toward either of these goals.

When exposing the water molecule to soft $\mathrm{x}$-ray radiation with an energy above the $\mathrm{O} 1 s$ ionization potential (IP, $539.9 \mathrm{eV}$ ) [1], an electron from the core orbital $\mathrm{O} 1 s$ can be ejected from the water molecule, which becomes ionized. The core-ionized state usually decays within a few fs via normal Auger transitions, which consist of an outer-shell

\footnotetext{
*christian.strahlman@mau.se
}

electron filling the core hole, paired with the emission of another outer-shell electron. This process usually leads to rapid fragmentation of the molecule.

Whereas molecular ionization dominates above the core ionization edge, other possibilities exist for the initial process. At certain incident photon energies, a core electron and a valence electron can be simultaneously excited to empty molecular or Rydberg orbitals, putting the system in a corevalence doubly excited state. At higher excitation energies, it is also possible for the emission of a core electron to be accompanied by the excitation of a valence electron, creating ionized shake-up states. Other above-threshold effects in molecules are shape resonances, where the core electron is temporarily trapped by a potential barrier. However, shape resonances are not present in isolated water molecules.

Several published studies have addressed these abovethreshold effects in gaseous water molecules. Shake-up levels have been studied in water by means of photoelectron spectroscopy with 650-eV incident photon energy [2]. The first of several shake-up limits was identified $17 \mathrm{eV}$ above the $\mathrm{O} 1 s$ ionization limit, which would translate to $557-\mathrm{eV}$ excitation energy. Kivimäki et al. [3] used photoabsorption and fluorescence spectroscopy to identify doubly excited 
states above the $\mathrm{O} 1 s$ threshold. Two broad structures were observed at photon energies 555-560 eV. In addition, Stolte et al. [4] have measured both negative and positive ion yields up to $552.5 \mathrm{eV}$. They observed an above-threshold resonance peak in the $\mathrm{O}^{-}$negative-ion yield of the water molecule near $550 \mathrm{eV}$, which was attributed to a doubly excited state. Guided by this result, Piancastelli et al. measured the resonant Auger spectrum at this resonance and observed that the spectrum displayed features originating from an Auger decay in an excited fragment [5]. This electronic decay was concluded to occur after ultrafast dissociation, where the core-valence doubly excited molecule dissociates on the same timescale as the Auger decay (a few fs).

Stråhlman et al. recently published a study [6] where the fragmentation processes of core-excited and core-ionized water molecules were studied with negative- and positive-ion coincidence (NIPICO) spectroscopy. In particular, this study showed a broad above-threshold resonance with a maximum at $\approx 556 \mathrm{eV}$ excitation energy in the NIPICO yield for the $\mathrm{H}^{-} / \mathrm{O}^{-}$pair. Stråhlman et al. argued that this broad peak had to be due to neutral doubly excited molecules decaying by resonant Auger decay and fragmenting to a $\mathrm{H}^{-} / \mathrm{O}^{+} / \mathrm{H}^{+}$ensemble. The study also showed that $\mathrm{O}^{-}$fragments were produced as a result of postcollision interaction (PCI) effects-namely, electron recapture. The study did not, however, reproduce the $\mathrm{O}^{-}$peak previously observed by Stolte et al. at $550 \mathrm{eV}$. The partly different results in the two studies prompted us to perform the present investigations with an improved instrument.

Complementing the previous experiments, we have performed quantum chemical calculations to further characterize the doubly excited states and confirm the assignment of the experimental features. We have used multiconfigurational self-consistent field calculations based on the restricted active space method including a perturbative treatment of dynamical correlation (RASPT2) [7,8] for an accurate description of the multielectron excitation process.

\section{METHODS AND INSTRUMENTATION}

The experimental work was performed using the Gas Phase Photoemission beamline at the Elettra synchrotron in Trieste, Italy. The beamline has an undulator light source. A spherical grating monochromator selects the photon energy, ranging from 13.5 to about $900 \mathrm{eV}$. Moreover, the radiation is linearly polarized. The beamline has been described in detail in previous studies [9].

For the experiment, we used an ion spectrometer assembly amended from the NIPICO instrument described in earlier works $[6,10,11]$. The pre-existing Wiley-McLaren-type [12] negative-ion TOF spectrometer was kept with identical performance as in our recent study [11]. A replacement for the positive-ion spectrometer was installed, as shown in Fig. 1. The new installation is, in fact, a time-of-flight spectrometer, although much shorter, to offer a high acceptance angle of positive fragments, which is especially useful to detect light hydrogen ions with a high transverse momentum. While the previous positive-ion spectrometer could accept all positive ions produced from the dissociation of water up to a cutoff kinetic energy of $2 \mathrm{eV}$ [6], the shorter instrument used in this work accepts positive ions up to a cutoff energy of $32 \mathrm{eV}$.

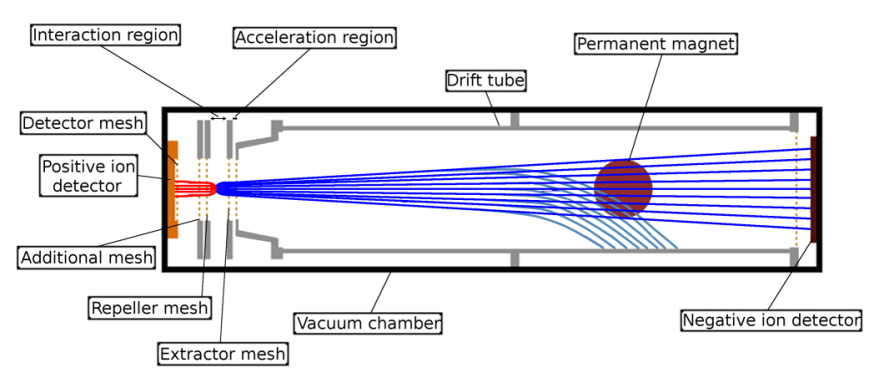

FIG. 1. Drawing of the NIPICO instrument. The positive ion (red) side has a very short drift tube. The negative ion (blue) side is unchanged compared to our previous setup [10]. Electrons (light blue) are deflected from the detector by a weak magnetic field.

The resolution was still sufficient to distinguish all possible fragment ions.

The positive-ion detector is a secondary electron multiplier (SEM-7M) from Baspik [13]. It is composed of an entrance mesh and two stacked multi channel plates (MCPs) connected in series, followed by an anode to pick up the electron signal. The signal is amplified and read by a time-to-digital converter, analogous to that of the negative-ion detector [10]. What is recorded is not a pure time-of-flight spectrum but rather an arrival-time-difference spectrum, where each peak is a signature of a negative- and positive-ion pair. The additional mesh inserted between the positive-ion detector and the extraction mesh enabled us to slightly modify the arrival times of the positive ions, hence the arrival-time difference. Such temporal tuning helps to distinguish real ion-ion coincidence peaks from spurious peaks. The drift tube potential in the negative-ion TOF spectrometer can be similarly tuned to distinguish real ion-ion coincidence peaks from electrons, photons, or spurious effects. Permanent magnets outside vacuum prevented electrons from reaching the negative-ion detector, although some were still recorded. In contrast to our previous work, only double coincidences (NIPICO) were recorded.

The pure water sample was prepared via freeze-pumpthaw cycling in order to remove all the residual gases from the sample container. The vapor pressure of the sample was sufficient to sustain an effusive jet through a gas needle close to the interaction region of the instrument. The valve used to regulate the gas flow was heated to $50^{\circ} \mathrm{C}$ so as to avoid condensation. The pressure in the chamber was $2.6 \times 10^{-6}$ mbar but was estimated to be 10-50 times higher in the interaction region. A photodiode was also placed in the path of the beam downstream of the interaction region in order to normalize the collected data to the beam intensity.

The detection rate was in the $10^{2} \mathrm{~Hz}$ range for the negativeparticle detector, and it was approximately ten times higher for positive ions. We estimated the contribution from electrons to be larger than the contribution from negative ions in the negative-ion spectrometer, even with the permanent magnets acting as a filter. However, since electrons are faster than any positive ion, these coincidences were filtered electronically.

The arrival-time difference coincidence spectrum measured with this setup is shown in Fig. 2. The different peaks were identified by measuring the spectra with different drift tube voltages and by comparing the results to the previous study [6]. Four NIPICO peaks were identified: $\mathrm{O}^{-} / \mathrm{H}^{+}$, 


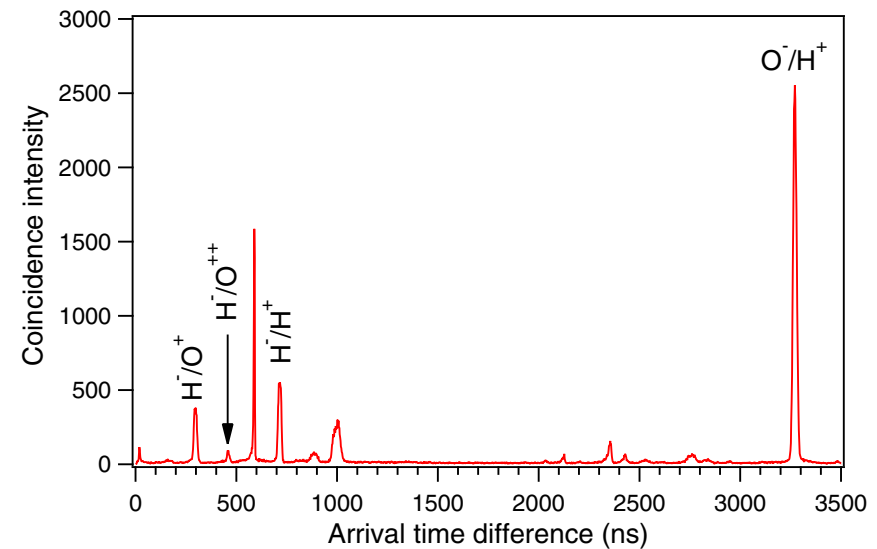

FIG. 2. Arrival-time difference spectrum recorded at $536.0 \mathrm{eV}$, which corresponds to the location of the $\left|\mathrm{O} 1 s^{-1} 2 b_{2}^{1}\right\rangle$ resonance below threshold. The four NIPICO peaks are indicated. The several spurious peaks are discussed in the text.

$\mathrm{H}^{-} / \mathrm{H}^{+}, \mathrm{H}^{-} / \mathrm{O}^{+}$, and $\mathrm{H}^{-} / \mathrm{O}^{++}$. The broad peaks located at 900 and $1000 \mathrm{~ns}$, as well as the sharp peaks at $580 \mathrm{~ns}$ and very close to $0 \mathrm{~ns}$, are spurious peaks that arise from the electronics or from cross-talk between the detectors. Those peaks, as well as the spurious peaks between 2000 and 3000 ns, are known from previous studies done with the original experimental setup [6], and we can confirm their spurious character using their insensitivity to the magnitude of the applied potentials.

\section{EXPERIMENTAL RESULTS}

Coincidences above the $\mathrm{O} 1 \mathrm{~s}$ ionization edge were measured by scanning the photon energy from 540 to $567 \mathrm{eV}$ in $0.4-\mathrm{eV}$ steps, collecting data for $600 \mathrm{~s}$ at each step. The intensity of the coincidence peak, normalized to the photon beam intensity, was plotted against photon energy, as illustrated in Fig. 3. The density of energy points is thus five times higher than in previous measurements with 2-eV steps [6], and the statistics are higher. Clearly, a lot more details are visible in this measurement. The improved detection of fast ions, namely $\mathrm{H}^{+}$, is especially significant for the $\mathrm{O}^{-} / \mathrm{H}^{+}$channel. The $\mathrm{O}^{-} / \mathrm{H}^{+}$channel is stronger than the $\mathrm{H}^{-} / \mathrm{O}^{+}$, opposite to earlier results [6]. We conclude that $\mathrm{O}^{-} / \mathrm{H}^{+}$was previously suppressed by the low detection rate of $\mathrm{H}^{+}$and appeared essentially featureless. This more reliable measurement shows a higher $\mathrm{O}^{-} / \mathrm{H}^{+}$yield and a much improved level of detail in general.

The present spectrum is characterised by some striking differences between, primarily, the $\mathrm{O}^{-} / \mathrm{H}^{+}$channel and the $\mathrm{H}^{-}$containing channels $\left(\mathrm{H}^{-} / \mathrm{H}^{+}, \mathrm{H}^{-} / \mathrm{O}^{+}\right.$, and $\left.\mathrm{H}^{-} / \mathrm{O}^{++}\right)$. The $\mathrm{O}^{-} / \mathrm{H}^{+}$spectrum has two major features. Just above threshold, the $\mathrm{O}^{-} / \mathrm{H}^{+}$ion pair yield rapidly diminishes, as can be seen in more detail in Fig. 4. According to Stolte et al. [4] and Stråhlman et al. [6], this is caused by postcollision interaction (PCI) effects, more specifically, electron recapture. Electron recapture arises when a core-ionized molecule undergoes Auger decay, and the slow $\mathrm{O} 1 s$ photoelectron is recaptured by the molecule as the removal of a fast Auger electron increases the attractive potential of the ionized molecule. The likelihood of electron recapture decreases as the energy increases above

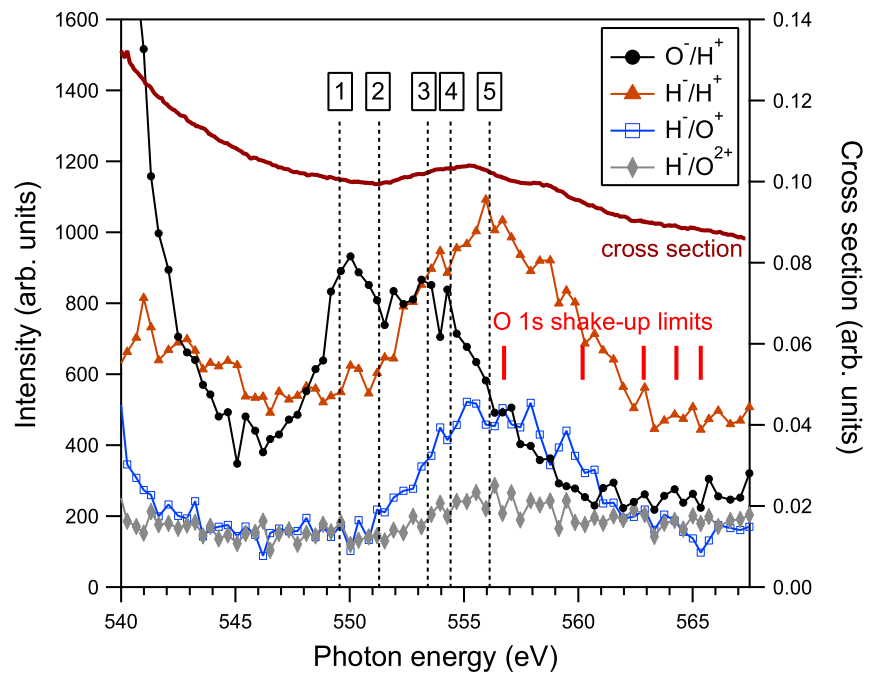

FIG. 3. Integrated NIPICO yields above the ionization threshold. The photoabsorption cross-section curve is reproduced from Ref. [3] and the shake-up limits were obtained from Ref. [2]. The calculated positions of the doubly excited states, indicated with dashed vertical lines and labeled with numbers $1-5$, are given in Table I.

the IP in an almost exponential fashion [14,15]. This effect is also seen for the $\mathrm{H}^{-} / \mathrm{O}^{+}$yield but not for the other $\mathrm{H}^{-}$ channels.

A structured feature can be seen in $\mathrm{O}^{-} / \mathrm{H}^{+}$between 548 and $557 \mathrm{eV}$, with (at least) two peaks at 550 and $554 \mathrm{eV}$. The first of these peaks was also observed by Stolte et $a l$. in the $\mathrm{O}^{-}$ noncoincident yield at $550 \mathrm{eV}$. However, the measurements

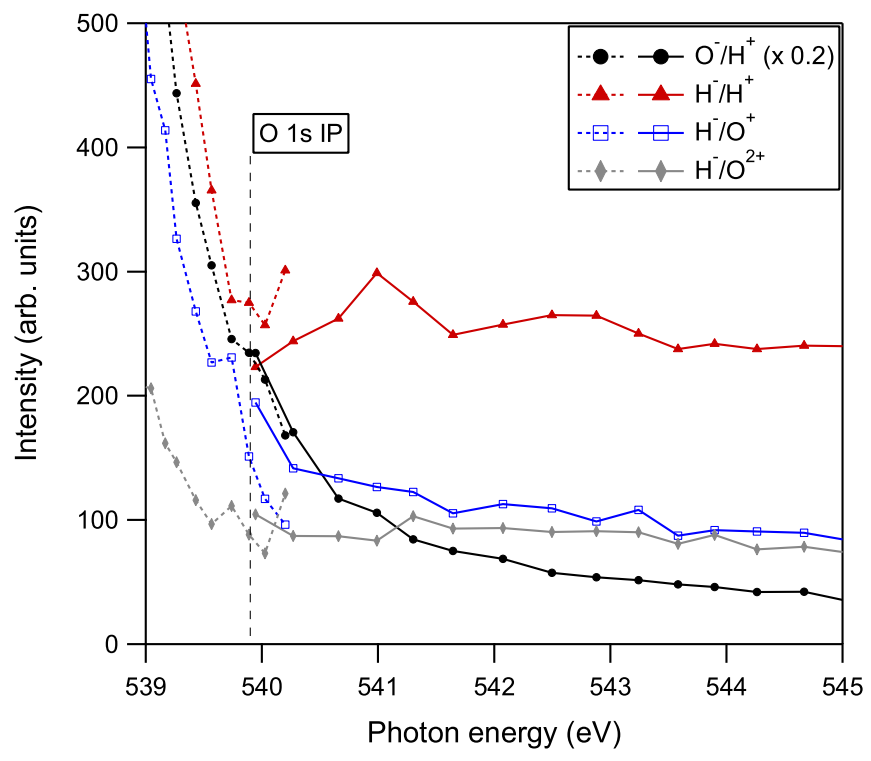

FIG. 4. NIPICO yields close to the O $1 s$ IP $(539.9 \mathrm{eV})$ for all observed negative- and positive-ion pairs. The $\mathrm{O}^{-} / \mathrm{H}^{+}$curves have been multiplied with 0.2 for easy comparison. The data displayed in this figure arise from two separate scans (indicated by solid and dashed lines), where the intensity of the synchrotron beam was increased three times for the higher energy measurement. This gives rise to an uncertainty in the normalization of the dashed lines to the solid lines. 
of the $\mathrm{O}^{-}$yield did not go above $552.5 \mathrm{eV}$, and they could therefore not observe the peak at $554 \mathrm{eV}$. As mentioned, the earlier study by Stråhlman et al. [11] lacked sufficient statistics and resolution to observe the $\mathrm{O}^{-} / \mathrm{H}^{+}$peak. Interestingly, the peak at $550 \mathrm{eV}$ does not overlap with any feature in the photoabsorption cross section, in the total ion yield, or in any fluorescent yield [3]. Therefore, its appearance seems to be significant only in the $\mathrm{O}^{-}$yield.

All $\mathrm{H}^{-}$containing channels have similar appearances above $545 \mathrm{eV}$, with a single broad feature peaking at $\sim 556 \mathrm{eV}$, in agreement with the observation of the $\mathrm{H}^{-} / \mathrm{O}^{+}$feature in our earlier study [11]. Contrary to the $\mathrm{O}^{-}$yield, the increase of the $\mathrm{H}^{-}$yield overlaps with the increase in photoabsorption cross section (and with increases in fluorescence yields [3]). Its right shoulder also extends above the two lowest shake-up limits.

\section{CAlCUlations}

The core excitations, including the doubly excited states, were derived from multistate RASPT2 calculations $[7,8]$ in MOLCAS [16]. Briefly, the calculations were performed in $C_{s}$ symmetry on an isolate water molecule at a geometry with $\mathrm{O}-\mathrm{H}$ distances of $0.9588 \AA$ and a bond angle of $104.21^{\circ}$ using the atomic natural orbital-relativistic and (semi-)core correlation (ANO-RCC) basis set [17] augmented with a Rydberg basis set. The RASPT2 calculations were performed with 11 active orbitals, of which the $\mathrm{O} 1 \mathrm{~s}$ orbital was put into the restricted active space (RAS3) space. This allowed us to control the occupancy of the core orbital and reach core-excited states. We used the same setup as in our previous calculations of potential energy surfaces for $x$-ray spectrum simulations of the water molecule [18] with a few exceptions: We used an imaginary shift of 0.15 au and performed state averaging over 40 states each in $A^{\prime}$ and $A^{\prime \prime}$ symmetry. Stability tests-varying the number of core-excited states, the active space, and the Rydberg basis-showed a variation of less than $\pm 0.1 \mathrm{eV}$ in the lowest core-valence doubly excited state presented in Table I: $\left|\mathrm{O} 1 s^{-1} 1 b_{1}^{-1} 4 a_{1}^{2}\right\rangle$. The $\left|\mathrm{O} 1 s^{-1} 3 a_{1}^{-1} 4 a_{1}^{2}\right\rangle,\left|\mathrm{O} 1 s^{-1} 1 b_{2}^{-1} 4 a_{1}^{2}\right\rangle$, and $\left|\mathrm{O} 1 s^{-1} 1 b_{1}^{-1} 2 b_{2}^{2}\right\rangle$ states also showed variations of about $\pm 0.1 \mathrm{eV}$. However,

TABLE I. The results from the RASPT2 calculations of the corevalence doubly excited states. Assignments, dipole moments, and energies of the ground and the lowest core-excited states are also given for comparison to the core-valence double excitations. For the core-valence doubly excited states, the additional energy required to excite the second electron is given in parentheses.

\begin{tabular}{cccc}
\hline \hline No. & State & $\begin{array}{c}\text { Dipole moment } \\
\text { (Debye) }\end{array}$ & $\begin{array}{c}\text { Energy } \\
(\mathrm{eV})\end{array}$ \\
\hline & $\mathrm{GS}$ & -1.8813 & 0 \\
& $\left|\mathrm{O} 1 s^{-1} 4 a_{1}^{1}\right\rangle$ & -0.6608 & 534.1 \\
1 & $\left|\mathrm{O} 1 s^{-1} 2 b_{2}^{1}\right\rangle$ & -0.9635 & 536.1 \\
2 & $\left|\mathrm{O} 1 s^{-1} 1 b_{1}^{-1} 4 a_{1}^{2}\right\rangle$ & 2.0407 & $549.6(15.4)$ \\
3 & $\left|\mathrm{O} 1 s^{-1} 3 a_{1}^{-1} 4 a_{1}^{2}\right\rangle$ & 2.1877 & $551.3(17.2)$ \\
4 & $\left|\mathrm{O} 1 s^{-1} 1 b_{2}^{-1} 4 a_{1}^{2}\right\rangle$ & 1.1472 & $553.4(19.3)$ \\
5 & $\left|\mathrm{O} 1 s^{-1} 1 b_{1}^{-1} 2 b_{2}^{2}\right\rangle$ & 1.4024 & $554.4(18.3)$ \\
\hline \hline & $\left|\mathrm{O} 1 s^{-1} 3 a_{1}^{-1} 2 b_{2}^{2}\right\rangle$ & 1.3974 & $556.1(20.0)$ \\
\hline
\end{tabular}

the $\left|\mathrm{O} 1 s^{-1} 1 b_{2}^{-1} 2 b_{2}^{2}\right\rangle$ state could not be assigned in the calculations using the larger active space and Rydberg basis. The variations increased with increasing excitation energywhich is seen already for the $\left|\mathrm{O} 1 s^{-1} 3 a_{1}^{-1} 2 b_{2}^{2}\right\rangle$, where the variation is $\pm 0.4 \mathrm{eV}$. Hence, we were not able to assign the $\left|\mathrm{O} 1 s^{-1} 1 b_{2}^{-1} 2 b_{2}^{2}\right\rangle$ state, which is highest in energy and mixes strongly with other core-excited states in our description.

Along with the difficulties of assigning the $\left|\mathrm{O} 1 s^{-1} 1 b_{2}^{-1} 2 b_{2}^{2}\right\rangle$ state, we notice that when including more Rydberg basis functions, it becomes increasingly difficult to reach and assign the $\left|\mathrm{O} 1 s^{-1} 3 a_{1}^{-1} 2 b_{2}^{2}\right\rangle$ state. Increasing the number of roots in the calculation of the core-excited states for a given basis leads to variations on the order of $0.2 \mathrm{eV}$ in that state. We also note that the larger Rydberg basis leads to a larger reference in the RASPT2 calculation of the $\left|\mathrm{O} 1 s^{-1} 3 a_{1}^{-1} 2 b_{2}^{2}\right\rangle$ state, but we anticipate that a significantly improved accuracy would require a larger active space.

From our calculations, we assign five doubly excited states in Table I. These states are formed by simultaneous excitation of an $\mathrm{O} 1 s$ core electron and an electron from the three highest occupied valence orbitals into the antibonding virtual orbitals, $4 a_{1}$ or $2 b_{2}$. Further, we only present the states with double occupation in the antibonding orbital, although doubly excited states with simultaneous occupation of both the $4 a_{1}$ and $2 b_{2}$ orbitals also occur at intermediate energies. Furthermore, while valence-Rydberg double excitations occur in the higher end of this energy interval, we do not have an adequate description of these states. Given the antibonding character of both $4 a_{1}$ and $2 b_{2}$ orbitals, we expect the doubly excited states (which have double occupied antibonding orbitals) to yield stronger gradients than the main $\left|\mathrm{O} 1 s^{-1} 4 a_{1}^{1}\right\rangle$ and $\left|\mathrm{O} 1 s^{-1} 2 b_{2}^{1}\right\rangle$ core excitations regardless of which valence orbital is depopulated. However, the measured fragmentation is due to core-excited state dynamics and dynamics in the final state of the Auger process, neither of which are modeled in the present study.

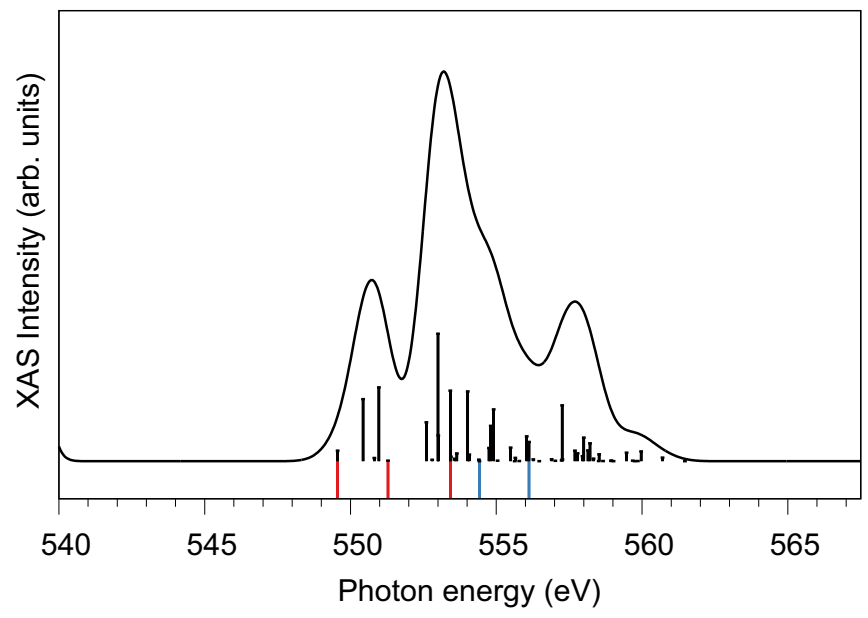

FIG. 5. The above-IP region of the calculated x-ray absorption spectrum. Both the discrete transitions and a sum spectrum convoluted with the Gaussian function (full-width-half-maximum of $1.3 \mathrm{eV}$ ) are shown. The core-valence doubly excited states presented in Table I are marked with red lines for the excitations into $4 a_{1}$ and with blue lines for the excitations into $2 b_{2}$. 
Figure 5 shows the calculated x-ray absorption spectrum above the O $1 s$ IP. In comparison to the measured photoabsorption cross section in Fig. 3, it shows a good overlap with observed features. The calculated peak at $550 \mathrm{eV}$ is, however, not observed in the experimental spectrum, indicating that these are weak transitions against the photoionization background. The calculations are designed to determine the energies of the core-valence double excitations, and we ascribe the disagreement to limitations in the description of the continuum states, which might artificially enhance the oscillator strength. We also note that the states with double excitations into $4 a_{1}$ are expected to be strongly dissociative and yield broad spectral features.

\section{DISCUSSION}

A defining feature of above-IP excitation is that a core electron can be kicked out of the molecule to create a highly excited core-ionized state $\mathrm{H}_{2} \mathrm{O}^{+}$. The core-ionized water molecules typically relax on the femtosecond timescale by emission of a Auger electron [19] or, with much lower probability, a fluorescent photon [20,21], which is a competing process. The result of the Auger process is a $\mathrm{H}_{2} \mathrm{O}^{++(*)}\left|\mathrm{val}^{-2}\right\rangle$ state (where "val" indicates any available valence orbital-2a $2 a_{1}, 1 b_{2}, 3 a_{1}$, or $1 b_{1}$-and $*$ denotes a valence-excited species). It has only one possible negative-ion fragmentation channel $\left(\mathrm{H}^{-} / \mathrm{O}^{2+} / \mathrm{H}^{+}\right)$. Such fragmentation would make a featureless contribution to the $\mathrm{H}^{-} / \mathrm{O}^{2+}$ and $\mathrm{H}^{-} / \mathrm{H}^{+}$yields in Fig. 3. At energies close to and just above IP, the normal Auger decay can also include recapture of the (slow) photoelectron into a virtual ("virt") orbital. The result is a singly charged $\mathrm{H}_{2} \mathrm{O}^{+*} \mid$ val $^{-2}$ virt $\left.{ }^{1}\right\rangle$ state where "virt" can be a $4 a_{1}, 2 b_{2}$, or Rydberg orbital. Such states can fragment into $\mathrm{O}^{-} / \mathrm{H}^{+} / \mathrm{H}, \mathrm{H}^{-} / \mathrm{O}^{+} / \mathrm{H}^{+}$, or $\mathrm{H}^{-} / \mathrm{O}^{2+} / \mathrm{H}$. The probability for electron recapture falls off as a close-to-exponential function as the photon energy increases away from the IP $[14,15]$. The detailed view in Fig. 4 shows an exponential trend for $\mathrm{H}^{-} / \mathrm{O}^{+}$ and $\mathrm{O}^{-} / \mathrm{H}^{+}$. While we acknowledge that the normalization between the two datasets is uncertain, the decaying trend is clearly visible in both. The exponential character of the $\mathrm{H}^{-} / \mathrm{O}^{+}$yield has not been observed previously. This observation confirms that both $\mathrm{O}^{-}$and $\mathrm{H}^{-}$fragments can be produced following electron recapture.

The $\mathrm{H}^{-} / \mathrm{H}^{+}$and $\mathrm{H}^{-} / \mathrm{O}^{2+}$, which can also be reached through a doubly charged parent molecule, do not show the same falling trend. The $\mathrm{H}^{-} / \mathrm{O}^{2+}$ remains almost flat at the IP, while $\mathrm{H}^{-} / \mathrm{H}^{+}$rather increases. This signifies the opening of the $\mathrm{H}^{-} / \mathrm{O}^{2+} / \mathrm{H}^{+}$fragmentation channel through normal Auger decay, which appears very close to the IP and extends toward higher energies.

In our previous study [11], we argued based on the recorded data that nonresonant radiative decay makes a contribution to the $\mathrm{H}^{-}$production above IP. We justified that claim based on the $\mathrm{H}^{-} / \mathrm{H}^{+}$yield increase just above threshold while other $\mathrm{H}^{-}$channels remained essentially flat. This study shows a less distinct rise in $\mathrm{H}^{-} / \mathrm{H}^{+}$. In addition, we can confirm the participation of $\mathrm{H}^{-}$in electron recapture. This makes it more plausible that the increase in $\mathrm{H}^{-} / \mathrm{H}^{+}$originates from the opening up of a normal Auger decay channel rather than radiative decay.

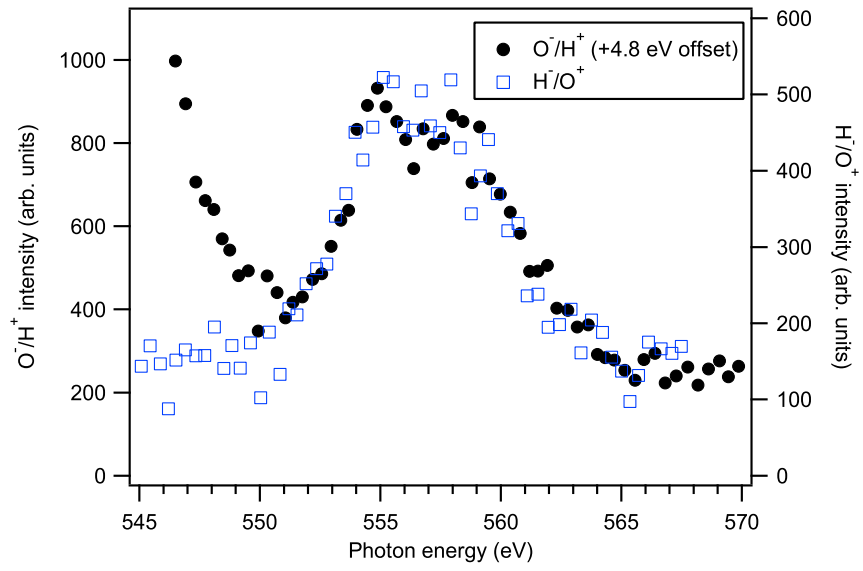

FIG. 6. Comparison between $\mathrm{H}^{-} / \mathrm{O}^{+}$and $\mathrm{O}^{-} / \mathrm{H}^{+}$with a $+4.8-\mathrm{eV}$ offset to the latter. Note that the curves have different intensity scales. The resonant features appear almost identical.

The origin of features in the NIPICO yields between 545 and $565 \mathrm{eV}$ is due to core-valence double excitations. The shake-up process, where one electron is emitted and another is promoted to a virtual orbital $\left|\mathrm{O} 1 s^{-1} \mathrm{val}^{-1} \operatorname{virt}^{1}\right\rangle$, can in principle contribute to negative-ion production above the first shake-up limit [2] but would only produce an increased yield above this photon energy. As such increases are not visible in Fig. 3, the shake-up contribution should be small compared to that of doubly excited states. The absence of shape resonances in water makes double excitations the only processes capable of producing the discrete features in the above-IP region. The core-valence double excitation involves one $\mathrm{O} 1 s$ core electron and one valence electron being promoted to virtual orbitals, which creates a neutral $\mathrm{H}_{2} \mathrm{O}^{* *} \mid \mathrm{O} 1 s^{-1}$ val $^{-1}$ virt $\left.^{2}\right\rangle$ state. Figure 3 shows a convincing overlap between the calculated energies of the doubly excited states and observed features. It is striking that the first $\mathrm{O}^{-} / \mathrm{H}^{+}$ feature at $550 \mathrm{eV}$ coincides with states 1-2 (see Table I), while the second feature appears to coincide with state 3 at $553 \mathrm{eV}$. States $1-3$ are all $\left|\mathrm{O} 1 s^{-1} \mathrm{val}^{-1} 4 a_{1}^{2}\right\rangle$ states, suggesting that $\mathrm{O}^{-} / \mathrm{H}^{+}$production is related to the doubly occupied $4 a_{1}$. Likewise, the channels containing $\mathrm{H}^{-}$have a similar overlap with $\left|\mathrm{O} 1 \mathrm{~s}^{-1} \mathrm{val}^{-1} 2 b_{2}^{2}\right\rangle$ states. This observation is particularly striking when the $\mathrm{H}^{-} / \mathrm{O}^{+}$and $\mathrm{O}^{-} / \mathrm{H}^{+}$yields are viewed together in Fig. 6. Our calculations in Table I predict a 4.8$\mathrm{eV}$ offset between $\left|\mathrm{O} 1 s^{-1} \mathrm{val}^{-1} 4 a_{1}^{2}\right\rangle$ and $\left|\mathrm{O} 1 s^{-1} \mathrm{val}^{-1} 2 b_{2}^{2}\right\rangle$ states. As we introduce the $4.8-\mathrm{eV}$ offset to the $\mathrm{O}^{-} / \mathrm{H}^{+}$curve, the similarity becomes obvious. Consequently, we suggest that $\mathrm{O}^{-}$fragments are produced mainly from $4 a_{1}^{2}$ states while $\mathrm{H}^{-}$fragments are produced mainly from $2 b_{2}^{2}$ states. An uncertainty in this interpretation is the $\left|\mathrm{O} 1 s^{-1} \mathrm{val}^{-1} 4 a_{1}^{1} 2 b_{2}^{1}\right\rangle$ states. They are likely to be populated in the same energy region, together with double excitations involving Rydberg orbitals. If these states were populated and participate in negativeion production, the result would be an increased yield of coincidences in the low end of the $\mathrm{H}^{-} / \mathrm{O}^{+}$feature and the high end of the $\mathrm{O}^{-} / \mathrm{H}^{+}$one. This would be expected to contribute asymmetrically to the yield curves, which is not evident in Fig. 6. At the low end of the $\mathrm{O}^{-} / \mathrm{H}^{+}$feature, no contribution other than $\left|\mathrm{O} 1 s^{-1} \mathrm{val}^{-1} 4 a_{1}^{2}\right\rangle$ is possible. 
The electrostatics of the water molecule in the different doubly excited electronic states was calculated and the molecular dipole moments are presented in Table I. However, this static picture does not explain the different trends in fragmentation. Hence, the nuclear dynamics and electronic decay would have to be simulated to model these processes, which is a tremendous theoretical effort beyond the scope of the current study.

The core-valence doubly excited state decays on a femtosecond timescale through fluorescent or resonant Auger emission, where the latter is expected to be much more probable. The resonant Auger decay can be a participator decay-where one of the electrons in a virtual orbital fills the core hole, creating $\mathrm{H}_{2} \mathrm{O}^{+*} \mid \mathrm{val}^{-2}$ virt $\left.^{1}\right\rangle$ states identical to the final states reached after spectator decay of resonant excitations below the $\mathrm{O} 1 s$ IP. The core-valence doubly excited state can also decay through spectator decay, where $\mathrm{a}_{2} \mathrm{O}^{+*} \mid$ val $^{-3}$ virt $\left.^{2}\right\rangle$ state is created. It has been argued that the spectator decay bears more weight than the participator decay for resonant states in water molecules [22]. It is reasonable to expect $\mathrm{H}_{2} \mathrm{O}^{+*} \mid \mathrm{val}^{-3}$ virt $\left.^{2}\right\rangle$ states to dominate the decay. It is likely that the $\mathrm{O}^{-} / \mathrm{H}^{+}$feature at $550 \mathrm{eV}$ originates from fragmentation of parent molecules with a $\left|\mathrm{val}^{-3} 4 a_{1}^{2}\right\rangle$ configuration. In order for an $\mathrm{O}^{-}$to be created, all electrons must remain attached to the oxygen atom while two protons are detached. Consequently, the data suggest that when the $4 a_{1}$ orbital becomes filled, the electrons prefer to remain with the oxygen atom as the molecule dissociates. The resonances at $550 \mathrm{eV}$ have not been observed in positive ion yields [4] and the resonances are not visible in the $\mathrm{x}$-ray absorption cross section. This suggests that the $\mathrm{O}^{-} / \mathrm{H}^{+} / \mathrm{H}^{+}$is a significant decay channel for these particular resonances.

For the $\mathrm{H}^{-}$-yielding resonances, centered at $556 \mathrm{eV}$, a similar argument can be made concerning the spectator Auger decay. States 4 and 5 (see Table I) decay into $\mathrm{H}_{2} \mathrm{O}^{+*}\left|\mathrm{val}^{-3} 2 b_{2}^{2}\right\rangle$. To create a $\mathrm{H}^{-}$fragment from dissociation, it is possible that both $2 b_{2}$ electrons remain attached to one hydrogen fragment while the valence holes are distributed among the remaining fragments. Alternatively, one $\mathrm{H}$ fragment keeps an electron in an occupied valence orbital and in addition captures one $2 b_{2}$ electron.

\section{CONCLUSION}

Experimental improvements in the detection of negativeand positive-ion coincidences combined with energy calculations of core-valence doubly excited states have allowed us to investigate the energy region above the $\mathrm{O} 1 s$ IP with increased resolution and efficiency. The study reveals that $\mathrm{H}^{-}$and $\mathrm{O}^{-}$ production has a strong electronic state dependence (i.e., different fragments appear at different excitation energies). With the aid of calculations, we assign these coincidence channels to certain doubly excited states. Our calculations and experimental results suggest that $\mathrm{O}^{-}$production and $\mathrm{H}^{-}$ production are associated with excitation to doubly occupied $4 a_{1}^{2}$ and $2 b_{2}^{2}$ states respectively.

Negative-ion creation through doubly excited states occurs against a background of negative-ion fragments created through normal Auger decay, where the doubly ionized parent molecule can dissociate into a $\mathrm{H}^{-} / \mathrm{O}^{2+} / \mathrm{H}^{+}$ensemble. The exponential fall off of the $\mathrm{O}^{-} / \mathrm{H}^{+}$and $\mathrm{H}^{-} / \mathrm{O}^{+}$yields shows that electron recapture causes $\mathrm{O}^{-} / \mathrm{H}^{+} / \mathrm{H}^{+}$and $\mathrm{H}^{-} / \mathrm{O}^{+} / \mathrm{H}^{+}$ fragmentation close to the $\mathrm{O} 1 s$ ionization potential.

\section{ACKNOWLEDGMENTS}

The authors thank A. Bjermo and the workshop at MAX IV Laboratory for their help with the design and construction of the instrument upgrade. We acknowledge Elettra Sincrotrone Trieste for providing beamtime (Proposal No. 20150229) and the staff at Elettra for their kind assistance. This work was supported by the Swedish Research Council. The quantum chemical (RASPT2) calculations were partially performed on resources provided by the Swedish National Infrastructure for Computing (SNIC).

The instrument upgrade was proposed by A.K. and designed by C.S. C.S., A.K., B.M., and R.R. commissioned the positive-ion spectrometer and performed the experiments at Elettra. B.M. made simulations of the instrument's performance and the initial data analysis. Those results have previously been published in B.M.'s master thesis [23], supervised by R.S. E.E. and M.O. made the calculations and simulations. C.S. and B.M. wrote the article with significant contributions from E.E., M.O., and A.K. All authors have given continuous feedback on the text.
[1] J. Schirmer, A. B. Trofimov, K. J. Randall, J. Feldhaus, A. M. Bradshaw, Y. Ma, C. T. Chen, and F. Sette, Phys. Rev. A 47, 1136 (1993).

[2] R. Sankari, M. Ehara, H. Nakatsuji, A. De Fanis, H. Aksela, S. L. Sorensen, M. N. Piancastelli, E. Kukk, and K. Ueda, Chem. Phys. Lett. 422, 51 (2006).

[3] A. Kivimäki, M. de Simone, M. Coreno, V. Feyer, E. M. García, J. Álvarez Ruiz, R. Richter, and K. C. Prince, Phys. Rev. A 75, 014503 (2007).

[4] W. C. Stolte, M. M. Sant'Anna, G. Öhrwall, I. Dominguez-Lopez, M. N. Piancastelli, and D. W. Lindle, Phys. Rev. A 68, 022701 (2003).
[5] M. N. Piancastelli, R. Sankari, S. Sorensen, A. De Fanis, H. Yoshida, M. Kitajima, H. Tanaka, and K. Ueda, Phys. Rev. A 71, 010703 (2005).

[6] C. Stråhlman, A. Kivimäki, R. Richter, and R. Sankari, J. Phys. Chem. A 120, 6389 (2016).

[7] P.-Å. Malmqvist, A. Rendell, and B. O. Roos, J. Phys. Chem. 94, 5477 (1990).

[8] P.-Å. Malmqvist, K. Pierloot, A. R. M. Shahi, C. J. Cramer, and L. Gagliardi, J. Chem. Phys. 128, 204109 (2008).

[9] K. C. Prince, R. R. Blyth, R. Delaunay, M. Zitnik, J. Krempasky, J. Slezak, R. Camilloni, L. Avaldi, M. Coreno, G. Stefani, C. 
Furlani, M. de Simone, and S. Stranges, J. Synchrotron Radiat. 5, 565 (1998).

[10] C. Stråhlman, R. Sankari, A. Kivimäki, R. Richter, M. Coreno, and R. Nyholm, Rev. Sci. Instrum. 87, 013109 (2016).

[11] C. Stråhlman, A. Kivimäki, R. Richter, and R. Sankari, Phys. Rev. A 96, 023409 (2017).

[12] W. C. Wiley and I. H. McLaren, Rev. Sci. Instrum. 26, 1150 (1955).

[13] Baspik Vladikavkaz Technological Center, http://www.baspik. com/eng/.

[14] W. Eberhardt, S. Bernstorf, H. W. Jochims, S. B. Whitfield, and B. Crasemann, Phys. Rev. A 38, 3808 (1988).

[15] G. Öhrwall, M. M. Sant'Anna, W. C. Stolte, I. DominguezLopez, L. T. N. Dang, A. S. Schlachter, and D. W. Lindle, J. Phys. B: At., Mol. Opt. Phys. 35, 4543 (2002).

[16] F. Aquilante, J. Autschbach, R. K. Carlson, L. F. Chibotaru, M. G. Delcey, L. De Vico, I. F. Galván, N. Ferré, L. M. Frutos, L. Gagliardi et al., J. Comput. Chem. 37, 506 (2015).
[17] B. O. Roos, R. Lindh, P.-Å. Malmqvist, V. Veryazov, and P.-O. Widmark, J. Phys. Chem. A 108, 2851 (2004).

[18] R. C. Couto, V. V. Cruz, E. Ertan, S. Eckert, M. Fondell, M. Dantz, B. Kennedy, T. Schmitt, A. Pietzsch, F. F. Guimarães, H. Ågren, F. Gel'mukhanov, M. Odelius, V. Kimberg, and A. Föhlisch, Nat. Commun. 8, 14165 (2017).

[19] H. Siegbahn, L. Asplund, and P. Kelfve, Chem. Phys. Lett. 35, 330 (1975).

[20] J. Nordgren, L. P. Werme, H. Ågren, C. Nordling, and K. Siegbahn, J. Phys. B: At. Mol. Phys. 8, L18 (1975).

[21] S. Kashtanov, A. Augustsson, Y. Luo, J.-H. Guo, C. Såthe, J.-E. Rubensson, H. Siegbahn, J. Nordgren, and H. Ågren, Phys. Rev. B 69, 024201 (2004).

[22] M. N. Piancastelli, A. Hempelmann, F. Heiser, O. Gessner, A. Rüdel, and U. Becker, Phys. Rev. A 59, 300 (1999).

[23] B. Moog, Positive-ion/negative-ion coincidence (PINICO) experiments on water, M.A. Thesis, Lund University, Lund, Sweden, 2017, http://lup.lub.lu.se/student-papers/record/8900836. 